\title{
OPEN Associations of pathological diagnosis and genetic abnormalities in meningiomas with the embryological origins of the meninges
}

Atsushi Okano ${ }^{1}$ Satoru Miyawaki ${ }^{1}$ Hiroki Hongo ${ }^{1}$ Shogo Dofuku ${ }^{1}$ Yu Teranishi Jun Mitsui ${ }^{2}$, Michihiro Tanaka ${ }^{3}$, Masahiro Shin ${ }^{1}$, Hirofumi Nakatomi ${ }^{1}$ \& Nobuhito Saito ${ }^{1}$

Certain driver mutations and pathological diagnoses are associated with the anatomical site of meningioma, based on which the meninges have different embryological origins. We hypothesized that mutations and pathological diagnoses of meningiomas are associated with different embryological origins. We comprehensively evaluated associations among tumor location, pathological diagnosis (histological type), and genetic alterations including AKT1, KLF4, SMO, POLR2A, and NF2 mutations and 22q deletion in 269 meningioma cases. Based on the embryological origin of meninges, the tumor locations were as follows: neural crest, paraxial mesodermal, and dorsal mesodermal origins. Tumors originating from the dura of certain embryologic origin displayed a significantly different pathological diagnoses and genetic abnormality ratio. For instance, driver genetic mutations with $A K T 1, K L F 4, S M O$, and POLR2A, were significantly associated with the paraxial mesodermal origin $\left(p=1.7 \times 10^{-10}\right)$. However, meningiomas with NF2-associated mutations were significantly associated with neural crest origin $\left(p=3.9 \times 10^{-12}\right)$. On analysis of recurrence, no difference was observed in embryological origin. However, POLR2A mutation was a risk factor for the tumor recurrence $\left(p=1.7 \times 10^{-2}\right.$, Hazard Ratio 4.08, 95\% Confidence Interval 1.28-13.0). Assessment of the embryological origin of the meninges may provide novel insights into the pathomechanism of meningiomas.

Meningiomas are the most common primary intracranial tumors accounting for $20 \%$ of all such tumors. Approximately $69 \%$ of meningiomas are benign (WHO grade I), while $29 \%$ are atypical (WHO grade II), and $2 \%$ are malignant (WHO grade III) ${ }^{1}$. Previous studies have suggested an association between meningioma location and histological grading, with non-skull-base meningiomas displaying more aggressive biological behavior ${ }^{2-6}$.

Molecular genetic investigations have revealed NF2 gene mutations in approximately $40-60 \%$ of sporadic meningiomas ${ }^{6,7}$. Recent studies have reported TRAF7, KLF4, AKT1, SMO, PIK3CA, and POLR2A mutations, all mutually exclusive of NF2 mutations ${ }^{8-13}$. In these reports, NF2 mutations and/or loss of chromosome 22 are predominant in meningiomas originating from the cerebral convexity and cerebellar dura and in the spinal canal $^{8,12,14,15}$. On comparing different skull-base locations, most non-NF2 meningiomas were located on the medial skull base, whereas those on the lateral and posterior skull base harbored NF2 mutations or loss of chromosome $22^{7,8,12,16-19}$. Thus, the gene mutations may be potentially associated with anatomical sites.

The meninges might have different embryological origins depending on the anatomical site. Numerous studies on meningeal development in humans strongly indicate three sources of embryogenesis: the neural crest, the paraxial mesoderm, and the dorsal mesoderm ${ }^{20-25}$. These differences in the embryological origin of the meninges are associated with the pathophysiology of various diseases ${ }^{26,27}$.

${ }^{1}$ Department of Neurosurgery, Faculty of Medicine, The University of Tokyo, 7-3-1 Hongo, Bunkyo-ku, Tokyo, Japan. ${ }^{2}$ Department of Molecular Neurology, Graduate School of Medicine, The University of Tokyo, 7-3-1 Hongo, Bunkyo-ku, Tokyo, Japan. ${ }^{3}$ Departments of Neuroendovascular Surgery, Kameda Medical Center, 929 Higashi-cho, Kamogawa, Chiba, Japan. ${ }^{\bowtie}$ email: miyawaki-tky@umin.ac.jp 


\begin{tabular}{|c|c|c|c|c|c|c|c|c|c|}
\hline & $\begin{array}{l}\text { General Cohort } \\
(\mathbf{n}=269)\end{array}$ & $\begin{array}{l}\operatorname{SMO}(\mathrm{n}=1 ; \\
0.3 \%)\end{array}$ & $\begin{array}{l}A K T 1(\mathrm{n}=28 \\
10.4 \%)\end{array}$ & $\begin{array}{l}K L F 4(\mathrm{n}=16 ; \\
5.9 \%)\end{array}$ & $\begin{array}{l}\text { POLR2A } \\
(\mathrm{n}=17 ; 6.3 \%)\end{array}$ & $\begin{array}{l}\text { NF2+22q loss } \\
(\mathrm{n}=87 ; 32.3 \%)\end{array}$ & $\begin{array}{l}\text { NF2 (n=19; } \\
7.1 \%)\end{array}$ & $\begin{array}{l}22 q \text { loss }(n=53 \\
19.7 \%)\end{array}$ & $\begin{array}{l}\text { Not detected } \\
(\mathrm{n}=48 ; \\
17.8 \%)\end{array}$ \\
\hline $\begin{array}{l}\text { Age }(\text { year }) \\
(\text { mean } \pm \text { SD) }\end{array}$ & $58.0 \pm 2.2$ & 59.9 & $57.3 \pm 2.4$ & $56.2 \pm 3.3$ & $51.6 \pm 3.2$ & $59.5 \pm 1.4$ & $53.7 \pm 3.0$ & $61.2 \pm 1.8$ & $58.7 \pm 0.9$ \\
\hline \multicolumn{10}{|l|}{ Sex } \\
\hline Female & $192(71.3 \%)$ & $0(0)$ & 20 (7.4\%) & $10(3.7 \%)$ & 17 (6.3\%) & $58(21.6 \%)$ & $16(5.9 \%)$ & $37(13.8 \%)$ & $34(12.6 \%)$ \\
\hline Male & $77(28.6 \%)$ & $1(0.3 \%)$ & $9(3.3 \%)$ & $6(2.2 \%)$ & $0(0)$ & $29(10.8 \%)$ & $3(1.1 \%)$ & $16(5.9 \%)$ & $14(5.2 \%)$ \\
\hline \multicolumn{10}{|l|}{ Localization } \\
\hline Anterior & $18(6.7 \%)$ & $1(0.3 \%)$ & $8(3.0 \%)$ & $0(0.3 \%)$ & $1(0.0 \%)$ & $2(0.7 \%)$ & $0(0)$ & $0(0)$ & $6(2.2 \%)$ \\
\hline Central & $76(28.3 \%)$ & $0(0)$ & $12(4.5 \%)$ & $11(4.1 \%)$ & $10(3.7 \%)$ & $17(6.3 \%)$ & $3(1.1 \%)$ & $8(3.0 \%)$ & $15(5.6 \%)$ \\
\hline Lateral & $25(9.3 \%)$ & $0(0)$ & $6(2.2 \%)$ & $4(1.5 \%)$ & $0(0)$ & $5(1.9 \%)$ & $0(0)$ & $4(1.5 \%)$ & $6(2.2 \%)$ \\
\hline Posterior & $28(10.4 \%)$ & $0(0)$ & $2(0.7 \%)$ & $0(0)$ & $2(0.7 \%)$ & $11(4.1 \%)$ & $2(0.7 \%)$ & $6(2.2 \%)$ & $5(1.9 \%)$ \\
\hline ST-med & $56(20.8 \%)$ & $0(0)$ & $0(0)$ & $1(0.3 \%)$ & $0(0)$ & $29(10.8 \%)$ & $5(1.9 \%)$ & $15(5.6 \%)$ & $6(2.2 \%)$ \\
\hline ST-ant/lat & $39(14.5 \%)$ & $0(0)$ & $0(0)$ & $0(0)$ & $0(0)$ & $14(5.2 \%)$ & $5(1.9 \%)$ & $16(5.9 \%)$ & $4(1.5 \%)$ \\
\hline ST-post/lat & $3(1.1 \%)$ & $0(0)$ & $0(0)$ & $0(0)$ & $0(0)$ & $0(0)$ & $0(0)$ & $1(0.3 \%)$ & $2(0.7 \%)$ \\
\hline $\begin{array}{l}\text { Cerebellar } \\
\text { tentorium }\end{array}$ & $24(8.9 \%)$ & $0(0)$ & $0(0)$ & $0(0)$ & $4(1.5 \%)$ & $9(3.3 \%)$ & $4(1.5 \%)$ & $3(1.1 \%)$ & $4(1.5 \%)$ \\
\hline \multicolumn{10}{|c|}{ Localization (embryological) } \\
\hline Neural crest & $144(53.5 \%)$ & $0(0)$ & $6(2.2 \%)$ & $5(1.9 \%)$ & $4(1.5 \%)$ & $57(21.2 \%)$ & $14(5.2 \%)$ & $38(14.1 \%)$ & $20(7.4 \%)$ \\
\hline $\begin{array}{l}\text { Paraxial meso- } \\
\text { derm }\end{array}$ & $97(36.1 \%)$ & $1(0.3 \%)$ & $20(7.4 \%)$ & $11(4.1 \%)$ & $11(4.1 \%)$ & $19(7.1 \%)$ & $3(1.1 \%)$ & $9(3.3 \%)$ & $23(8.6 \%)$ \\
\hline $\begin{array}{l}\text { Dorsal meso- } \\
\text { derm }\end{array}$ & $28(10.4 \%)$ & $0(0)$ & $2(0.7 \%)$ & $0(0)$ & $2(0.7 \%)$ & $11(4.1 \%)$ & $2(0.7 \%)$ & $6(2.2 \%)$ & $5(1.9 \%)$ \\
\hline \multicolumn{10}{|l|}{ WHO grade } \\
\hline I & $243(90.3 \%)$ & $1(0.3 \%)$ & $27(10.4 \%)$ & $16(5.9 \%)$ & $17(6.3 \%)$ & $76(28.3 \%)$ & $18(6.7 \%)$ & $43(16.0 \%)$ & $45(16.7 \%)$ \\
\hline II & $26(9.7 \%)$ & $0(0)$ & $1(0.3 \%)$ & $0(0)$ & $0(0)$ & $11(4.1 \%)$ & $1(0.3 \%)$ & $10(3.7 \%)$ & $3(1.1 \%)$ \\
\hline III & 0 & 0 & 0 & 0 & 0 & 0 & 0 & 0 & 0 \\
\hline $\begin{array}{l}\text { MIB-1 LI } \\
(\text { mean } \pm \text { SD })\end{array}$ & $3.1 \pm 0.2$ & 3 & $2.1 \pm 0.7$ & $1.7 \pm 0.9$ & $1.2 \pm 0.9$ & $3.9 \pm 0.4$ & $2.6 \pm 0.8$ & $3.6 \pm 0.5$ & $2.8 \pm 0.5$ \\
\hline \multicolumn{10}{|l|}{ Simpson grade } \\
\hline 1 & 50 & 0 & 5 & 1 & 0 & 15 & 6 & 15 & 8 \\
\hline 2 & 115 & 0 & 15 & 5 & 9 & 35 & 9 & 18 & 24 \\
\hline 3 & 30 & 1 & 1 & 4 & 2 & 9 & 3 & 7 & 3 \\
\hline 4 & 53 & 0 & 7 & 6 & 6 & 16 & 1 & 7 & 11 \\
\hline 5 & 3 & 0 & 0 & 0 & 0 & 2 & 0 & 1 & 0 \\
\hline $\begin{array}{l}\text { Follow up } \\
\text { (years) }\end{array}$ & $4.2 \pm 0.2$ & 1.3 & $3.3 \pm 0.7$ & $4.3 \pm 1.0$ & $3.8 \pm 1.0$ & $3.8 \pm 0.4$ & $4.0 \pm 0.9$ & $4.3 \pm 0.5$ & $5.1 \pm 0.6$ \\
\hline Recurrence & $51(19.0 \%)$ & $1(100 \%)$ & $3(10.3 \%)$ & $2(12.5 \%)$ & $5(29.4 \%)$ & $22(25.6 \%)$ & $2(10.5 \%)$ & $10(19.2 \%)$ & $6(12.5 \%)$ \\
\hline
\end{tabular}

Table 1. Patient characteristics with clinical, genetic and histological features. $S D$ indicates standard deviatation, ST-med supra-tentorial-medial, ST-ant/lat supra-tentorial-antero-lateral, ST-post/lat supratentorial-postero-lateral, $L I$ labelling index.

We hypothesized that driver mutations and meningioma-related pathologies are associated with the meningeal origin. We modified the concept of tumor location in accordance with the embryological origin of different meningeal parts to verify this hypothesis. Thereafter, we comprehensively evaluated the associations between tumor location (embryological origin), pathological diagnosis (histological type), and driver mutations including $A K T 1, K L F 4, S M O, P O L R 2 A$, and NF2 mutations. Finally, we evaluated the factors affecting tumor recurrence, such as clinical parameters, embryological origins, pathological diagnosis and genetic mutations.

\section{Results}

Patient characteristics. A total of 499 meningioma patients who had undergone surgery at the University of Tokyo Hospital, Tokyo, Japan, between January 2000 and June 2017 were included herein. Among them, 269 patients adhered to the study inclusion criteria. This study included 192 women (71\%) and 77 men (29\%). The patient mean age was 58.0 years (range; $0.1-81.2$ years) and mean follow-up duration was 50.4 months (range; 1-199) (Table 1).

Pathological diagnosis and embryological origins of the meninges. Tablel provides raw data regarding tumor locations, genetic status and clinico-histopathological features.

Figure $1 \mathrm{~A}-\mathrm{C}$ shows a schematic representation of the spatial distribution of the embryological origins of the meninges based on previous reports. Dura with neural crest origin is purple, that originating from the paraxial mesoderm is green, and that originating from the dorsal mesoderm origin is blue. Herein, 144 cases 

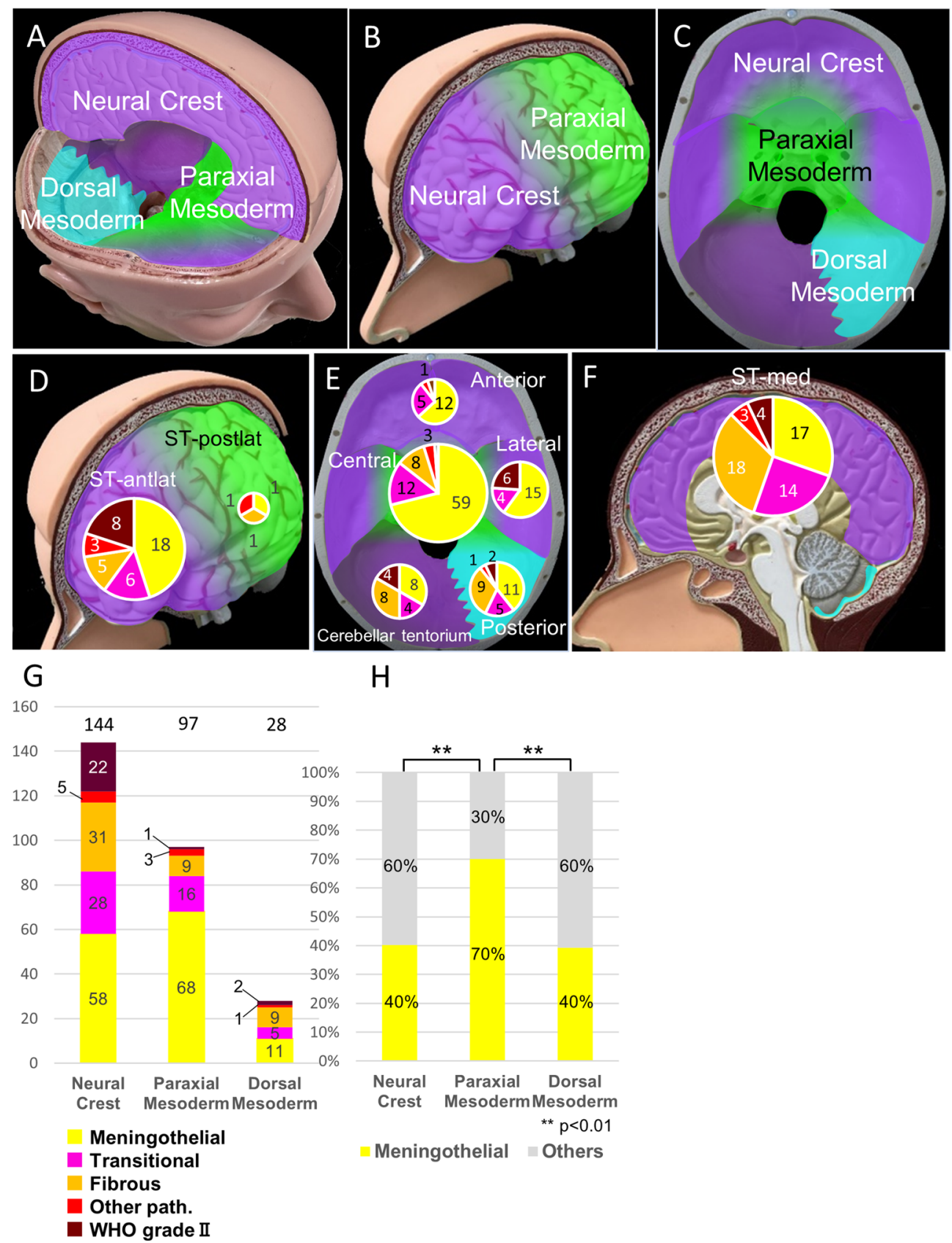

$\mathrm{H}$

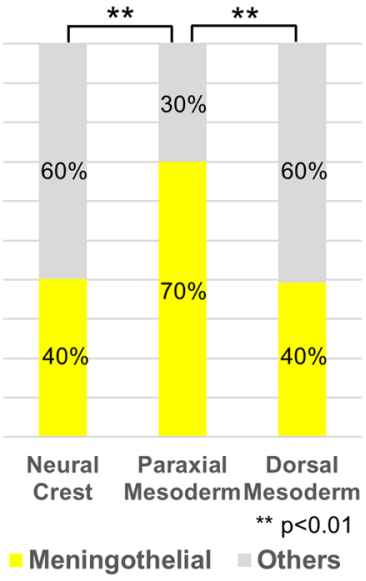

Figure 1. Pathological diagnosis according to embryologically classified anatomical location of meningiomas. (A-C) Embryological origins of the intracranial meninges. (D) Pathological distribution at ST-ant/lat and ST-post/lat. Each pie chart provides a breakdown of the number of each pathological diagnosis. The size of the pie charts reflects the total number of cases of this type of lesion. (E) Pathological distribution at the skullbase. The numbers indicate the number of cases for each location. The sizes of the pie chart indicate the total number of cases for each location. (F) Pathological distribution at ST-med. The number indicates the number of cases for each location. The sizes of the pie chart indicate the total number of cases for each location. (G) The number of pathological diagnoses for each embryologically classified tumor. The bar graph shows the number of pathological diagnoses for each embryologically classified tumor. $(\mathbf{H})$ Comparison of the ratio of meningothelial meningioma among embryologically classified tumors. The ratio of meningothelial meningioma is significantly higher in tumors derived from the paraxial mesoderm rather than the neural crest or dorsal mesoderm. $(p<0.01$, statistically significant on the chi-square test with Bonferroni correction). ST-ant/lat, supra-tentorialantero-lateral; ST-post/lat, supra-tentorial-postero-lateral; ST-med, supra-tentorial-medial. Figure generated using Microsoft PowerPoint 2016, https://www.microsoft.com. 


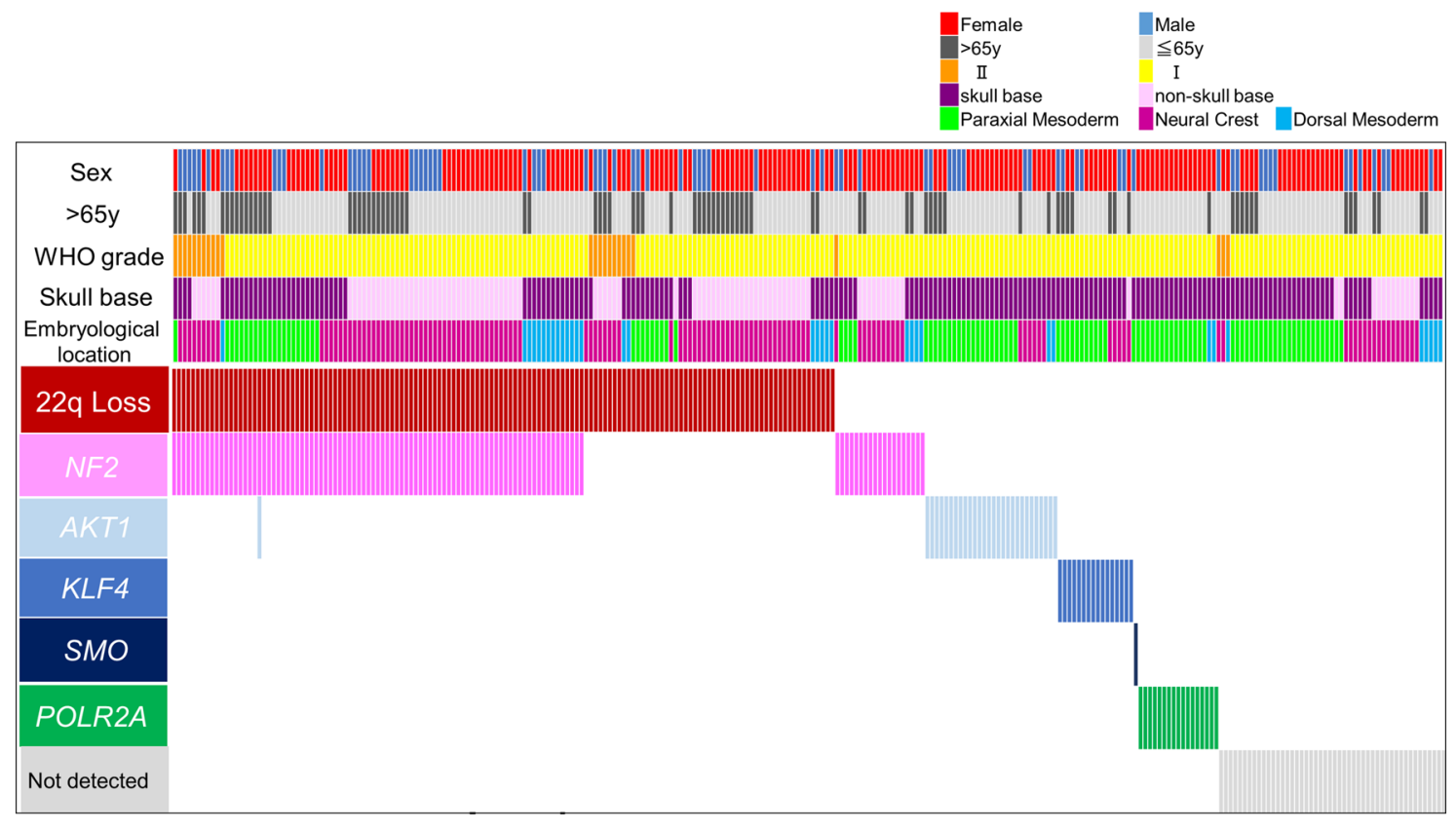

Figure 2. Overall genetic status and clinical characteristics. NF2 mutation and/or 22q loss, AKT1, KLF4, $P O L R 2 A$ and SMO mutation are mutually exclusive and only one tumor harbored NF2 and AKT1 mutations. The remaining 48 cases were defined as "Not detected," implying that none of these mutations were detected. 269 cases are depicted. Figure generated using Microsoft PowerPoint 2016, https://www.microsoft.com.

were included in the neural crest group, 97 cases in the paraxial mesoderm group, and 28 cases in the dorsal mesoderm group (Table 1).

Pathological diagnosis revealed WHO grade I meningioma in 243 tumors (90.3\%), including 135 of meningothelial, 49 of transitional, and 49 of fibrous types. WHO grade II tumors were detected in 26 cases (9.7\%), while no cases presented WHO grade III tumors. The proportion of fibrous meningiomas was relatively higher in the ST-med and posterior skull base. WHO grade II meningiomas were more frequent at ST-ant/lat, ST-med, lateral, and posterior, and meningothelial meningiomas were more frequent at the skull base, especially in the "Central" and "Anterior" regions (Fig. 1D-F). One of the reasons for the small number of WHO grade II cases herein may be that our cohort has a high proportion of skull base meningiomas. In fact, 171 of 269 (63.6\%) tumors were located at the skull base.

Regarding the association between pathological diagnosis and embryological origins of the meninges, the proportion of meningothelial meningiomas was significantly higher in patients with lesions originating from the paraxial mesoderm rather than from the neural crest $\left(p=5.5 \times 10^{-6}\right)$ and the dorsal mesoderm $\left(p=2.9 \times 10^{-3}\right)$ (Fig. $1 \mathrm{G}, \mathrm{H}$ ). However, the proportion of fibrous meningiomas was significantly higher among patients with lesions originating from the neural crest rather than the paraxial mesoderm $(p=0.01)$ (Supplemental Fig. 1A). The proportion of WHO grade II meningiomas was higher among patients with lesions originating from the neural crest rather than from the paraxial mesoderm $\left(p=1.4 \times 10^{-4}\right)$ (Supplemental Fig. 1B).

Genetic mutations and embryological origins of the meninges. Mutations detected in the 269 cases were localized at AKT1 in 29 cases, KLF4 in 16 cases, SMO in one case, POLR2A in 17 cases, with NF2 and $22 \mathrm{q}$ loss in 87 cases, and NF2 only in 19 cases and 22q loss only in 53 cases (Fig. 2). Representative cases and the results of Sanger sequencing of each mutation are illustrated in Supplemental Fig. 2. These mutations were mutually exclusive and only one tumor harbored NF2 and AKT1 mutations. The remaining 48 cases were defined as "Not detected," implying that none of these mutations were detected (Fig. 2).

Tumors in almost all patients harboring any one of these four mutations were present along the skull base, with the exception of one patient harboring a KLF4 mutation wherein the tumor was located in the ST-post/ lat region. Notably, only one meningioma with an $S M O$ mutation had an anterior location; however, numerous tumors harboring KLF or POLR2A mutations had a central location. Furthermore, numerous tumors harboring an AKT1 mutation had anterior and central locations (Fig. 3A-C).

Regarding the association between genetic alterations and embryological origins of the meninges, Fig. 3D shows the numbers of patients harboring each genetic mutation at each embryologically classified region. Figure $3 \mathrm{E}$ shows the numbers of patients harboring each genetic mutation at the skull base or supra tentorial. In particular, $A K T 1, K L F 4, S M O$, or POLR2A mutations were significantly more frequent in meningiomas of paraxial mesodermal origin than in those of neural crest $\left(p=1.7 \times 10^{-10}\right)$ and dorsal mesodermal origin $\left(p=3.0 \times 10^{-4}\right)$ (Fig. 3F). AKT1, KLF4, SMO, or POLR2A mutations were significantly more frequent in meningiomas of skullbase lesions than in those of supra-tentorial lesions $\left(p=8.3 \times 10^{-11}\right)$ (Fig. 3G). Regarding patients harboring NF2 mutations and/or $22 \mathrm{q}$ loss, these mutations were more frequent in meningiomas of neural crest than in those 


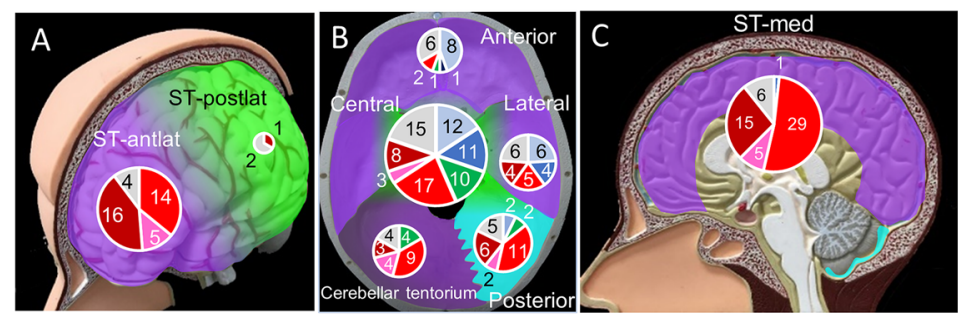

$\square A K T 1 \square K L F 4 \square S M O \square P O L R 2 A \square N F 2+22 q$ loss $\square$ NF2 $\square 22 q$ loss $\square$ Not detected



\section{$\mathrm{E}$}

$\mathrm{F}$

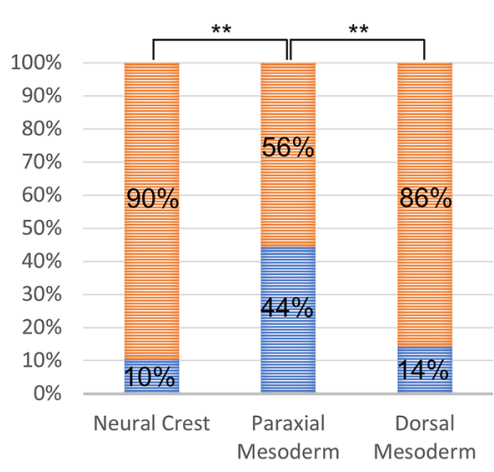

aKT1, KLF4, SMO, POLR2A "Others

** $p<0.01$

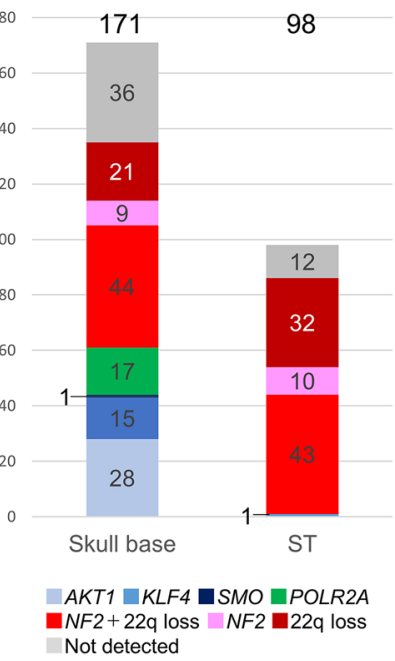

G

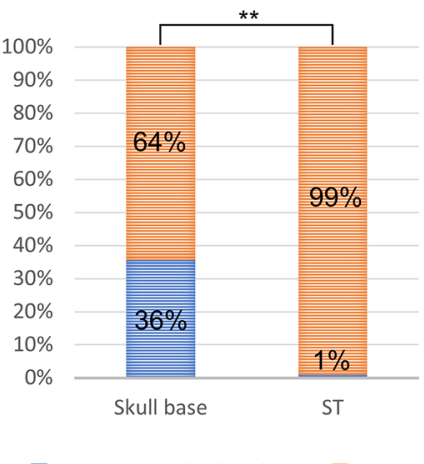

口AKT1, KLF4, SMO, POLR2A $\square$ Others

** $p<0.01$

Figure 3. Genetic mutations according to embryologically classified anatomical location of meningiomas. (A) Distribution of genetic mutations at ST-ant/lat and ST-post/lat. Each pie chart shows the breakdown of the number of patients harboring each type of mutation. The sizes of the pie charts reflect the total number of the patients with each type of lesion. (B) Distribution of mutations in skull-base tumors. The numbers indicate the number of patients harboring tumors at each location. The sizes of the pie chart indicate the total number of patients with tumors at different locations. (C) Distribution of mutations at ST-med. The number indicates the number of patients with tumors at each location. The sizes of the pie chart indicate the total number of patients harboring tumors at each location. (D) The number of patients harboring mutations at each embryologically classified location. (E) The number of patients harboring tumors at the skull base or Supra tentorial area, with mutations. The bar graph shows the numbers of patients with each type of mutation per location. (F) Comparison of the ratio of patients harboring AKT1, KLF4, SMO, or POLR2A mutations among embryologically classified locations. The ratio of patients harboring AKT1, KLF4, SMO, or POLR2A mutations was higher for tumors originating from the paraxial mesoderm location rather than the neural crest or dorsal mesoderm ( $p<0.01$, significant on the chi-square test with Bonferroni correction). (G) Comparison of the ratio of patients harboring AKT1, KLF4, SMO, or POLR2A mutations between skull-base and supra-tentorial tumors. The ratio of patients harboring $A K T 1, K L F 4, S M O$, or POLR2A mutations was higher for skull-base tumors rather than supra tentorial tumors ( $p<0.01$, significant on the chi-square test with Bonferroni correction). ST-ant/lat, supra-tentorial-antero-lateral; ST-post/lat, supra-tentorial-postero-lateral; ST-med, supra-tentorialmedial. Figure generated using Microsoft PowerPoint 2016, https://www.microsoft.com. 


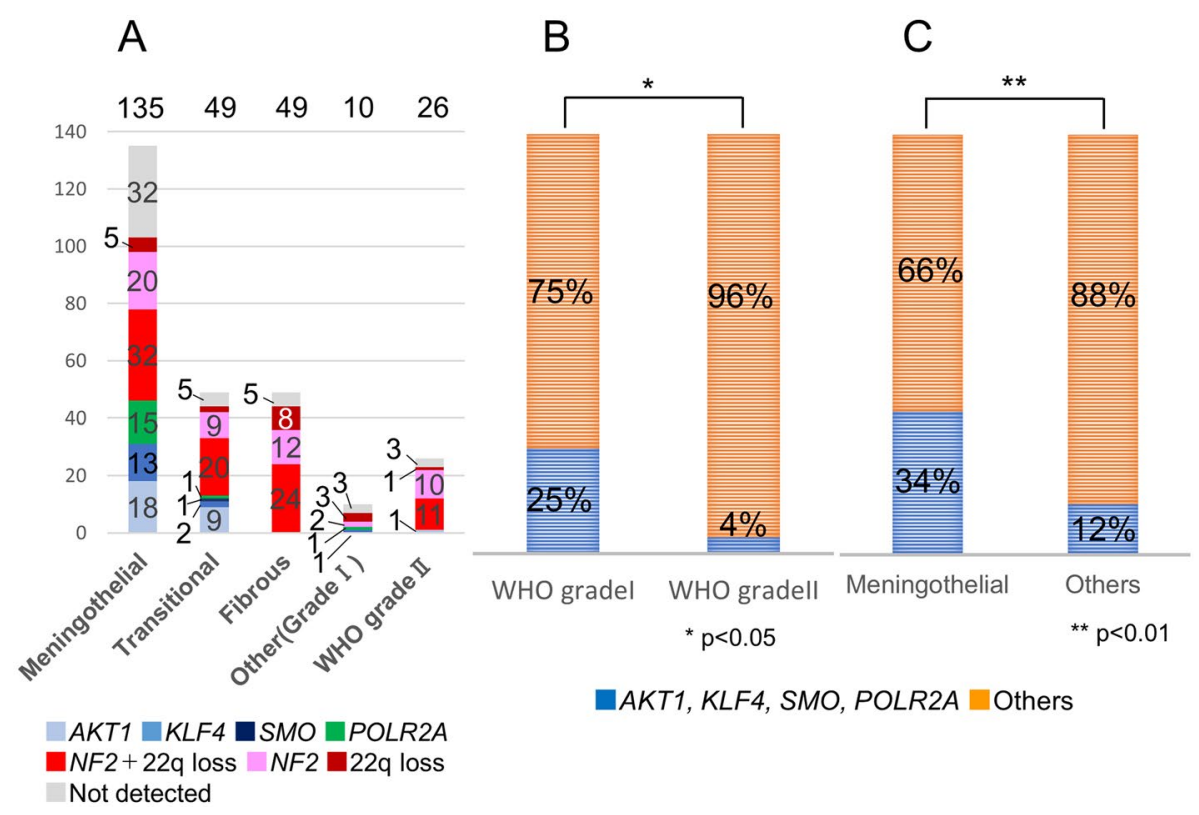

Figure 4. Distribution of genetic mutations among different pathological diagnosis. (A) The number of patients harboring mutations for each pathological diagnosis. (B) Comparison of the ratio of patients harboring AKT1, KLF4, SMO, or POLR2A mutations between WHO grade I and WHO grade II tumors. The ratio of patients harboring AKT1, KLF4, SMO, or POLR2A mutations was significantly higher for WHO grade I than for WHO grade II tumors $(p<0.05$, significant on the chi-square test with Bonferroni correction). (C) Comparison of the ratio of patients harboring $A K T 1, K L F 4, S M O$, or POLR2A mutations between meningothelial meningioma and other pathologies. The ratio of patients harboring AKT1,KLF4, SMO, or POLR2A mutations was higher for meningothelial meningioma than for other pathologies $(p<0.01$, significant on the chi-square test with Bonferroni correction).

of paraxial mesodermal origin $\left(p=3.9 \times 10^{-12}\right)$ and more frequent in those of neural crest rather than dorsal mesodermal origin $\left(p=5.0 \times 10^{-5}\right)$ (Supplemental Fig. 3).

Genetic mutations and pathological diagnosis. The number of patients harboring each mutation based on pathological diagnosis is indicated in a bar graph in Fig. 4A. AKT1, KLF4, SMO, or POLR2A mutation was significantly more frequent in WHO grade I meningioma than in WHO grade II meningioma $(p=0.01)$ (Fig. 4B), and any one of these four mutations was more frequently associated with meningothelial histological types than with other pathological types. $\left(p=1.6 \times 10^{-5}\right)$ (Fig. $\left.4 \mathrm{C}\right)$. As an exception, one patient presented with a psammomatous meningioma with a POLR2A mutation (Supplemental Fig. 4), and another patient presented with an angiomatous meningioma with a KLF4 mutation, while yet another patient presented with an atypical meningioma with an AKT1 mutation. However, the 49 patients with fibrous and 25 patients with WHO grade II meningiomas did not harbor these four mutations, except one patient. Forty-four of the 49 patients $(89.8 \%)$ with fibrous type and 22 of the 25 patients $(88.0 \%)$ with WHO grade II meningiomas harbored an NF2 mutation or $22 \mathrm{q}$ loss. Among WHO grade I tumors, NF2 mutations or $22 \mathrm{q}$ loss were significantly more frequent in fibrous meningioma than in other pathological types $\left(p=1.3 \times 10^{-7}\right)$ (Supplemental Fig. 5).

Figure 5 summarizes the anatomogenetic characteristics of each type of pathology.

Effects on the recurrence rates of meningioma. In this study, embryologically classified tumor locations were not prognostic factors both with the log-rank test $(p=0.86)$ (Fig. 6A) and a Cox proportional hazards model (Table 2). However, Kaplan-Meier curves comparing tumor recurrence among patients harboring different mutations and those without these mutations revealed that the presence of a mutation may potentially play a predictive role (Fig. $6 \mathrm{~B}-\mathrm{E}$ ). Patients harboring POLR2A mutations experienced tumor recurrence with a high rate of $29.4 \%$ (Table 1). This group displayed a significant difference on the log-rank test $(p=0.05)$ (Fig. 6D). Furthermore, we analyzed the factors associated with tumor recurrence through a Cox proportional hazards model (Table 2). Multivariate analysis was performed using factors with a $p$ value of $\leq 0.20$ on univariate analysis. In the multivariate model, WHO grade II $\left(p=1.2 \times 10^{-4}\right.$, Hazard Ratio [HR] 4.99, 95\% Confidence Interval [CI] $2.20-11.3)$, Simpson grade $1-3\left(p=1.9 \times 10^{-6}\right.$, HR $0.21,95 \%$ CI $\left.0.11-0.39\right)$, and POLR2A mutation $\left(p=1.7 \times 10^{-2}\right.$, HR 4.08, 95\% CI 1.28-13.0) were associated with tumor recurrence (Table 2).

Five of 17 patients harboring a POLR2A mutation experienced tumor recurrence. All five patients underwent partial tumor resection during initial surgery because the tumor was located at the central skull base. Nevertheless, the presence of the POLR2A mutation served as a determinant of tumor recurrence independent of Simpson grade 1-3. The follow-up duration among patients harboring the POLR2A mutation did not markedly differ 

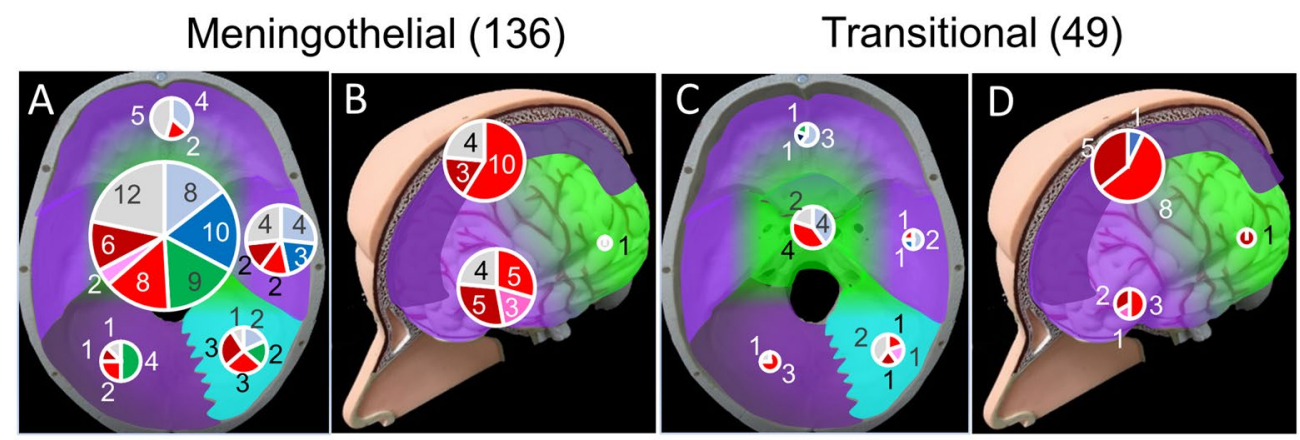

Fibrous(49)
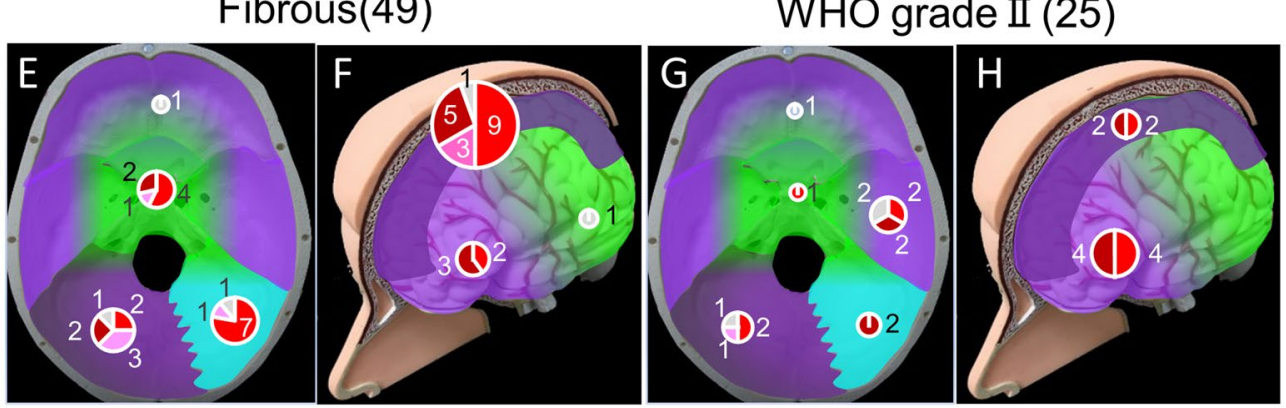

$A K T 1 \square K L F 4 \square S M O \square P O L R 2 A \square$ NF2 + 22q loss $\square$ NF2 $\square 22 q$ loss $\backsim$ Not detected

Figure 5. Associations between genetic mutations, histological type, and tumor locations. Each pie chart shows the breakdown of patients harboring each type of mutation. The size of pie charts reflects the total number of patients harboring each type of lesion. (A) Meningothelial tumor in the skull base. (B) Meningothelial tumor in the supra tentorial region. (C) Transitional tumor in the skull base. (D) Transitional tumor in the supra tentorial region. (E) Fibrous tumor in the skull base. (F) Fibrous tumor in the supra tentorial region. (G) WHO grade II tumor in the skull base. (H) WHO grade II tumor in the supra tentorial region. Figure generated using Microsoft PowerPoint 2016, https://www.microsoft.com.

from that of patients harboring other mutations. Furthermore, all patients harboring the POLR2A mutation were followed-up at our institution. We examined the effect of the POLR2A mutation among skull-base WHO grade I tumors and found that patients harboring the POLR $2 A$ mutation had a significantly worse prognosis $\left(p=8.9 \times 10^{-3}\right)$ (Supplemental Fig. 6). Furthermore, we analyzed the factors associated with tumor recurrence through a Cox proportional hazards model in this group (Supplemental Table 1). Multivariate analysis was performed using factors with a $p$-value of $\leq 0.20$ on univariate analysis. In the multivariate model, Simpson grade $1-3\left(p=1.5 \times 10^{-3}\right.$, HR $0.25,95 \%$ CI $\left.0.11-0.59\right)$, MIB-1 LI $\geq 3(p=0.01$, HR $3.26,95 \%$ CI $1.27-8.40)$ and POLR2A mutation ( $p=0.04$, HR $2.80,95 \%$ CI $1.16-9.53)$ were significantly associated with tumor recurrence. Hence, the POLR2A mutation may be a potentially useful predictor of tumor recurrence in skull-base WHO grade I meningiomas.

\section{Discussion}

This study comprehensively illustrates the anatomopathological association among driver mutations in meningiomas. Associations among anatomical locations, pathological diagnosis, and driver mutations were consistent with those reported previously ${ }^{12,16,19}$. However, this study reveals novel findings about the associations of the embryological origins of the meninges at different anatomical locations with genetic backgrounds and pathological diagnoses. Moreover, this study reports that the POLR2A mutation may serve as a potential marker for meningiomas with poor prognoses.

This study defined the locations of tumor origins on the basis of existing knowledge of the embryological origin of leptomeninges. Meningeal convexities originate from the skeleto-genesis layer immediately adjacent to them ${ }^{20}$. Furthermore, the calvaria develops from the embryonic head mesenchyme surrounding the brain, similar to the meninges; hence, its progenitors are presumably included in the primary menin $\mathrm{x}^{21}$, suggesting a similar origin of the meninges and bones. In mammals, the skull vault is constructed from embryogenic tissues of the neural crest and mesoderm ${ }^{22,23}$. The coronal suture separates the neural-crest-derived frontal bone from the paraxial mesodermal parietal bone ${ }^{22,25}$. Therefore, the anterior region of the meningeal convexity is expected to originate from the neural crest and the posterior region from the paraxial mesoderm.

Falx cerebri and cerebellar tentorium are derived from the neural crest ${ }^{20,22}$. Differences in the prechordal plate differentiates into the neural crest and forms the cerebellar tentorium adjacent $\mathrm{it}^{28}$. The neural crest forms the anterior region of the falx. After the posterior region of the falx is formed, both regions interact.

Regarding skull-base meninges, McBratney-Owen et al. reported that in rats, the anterior cranial base is derived from the neural crest, the posterior cranial base is derived from the mesoderm and the sphenoid bone is 
A

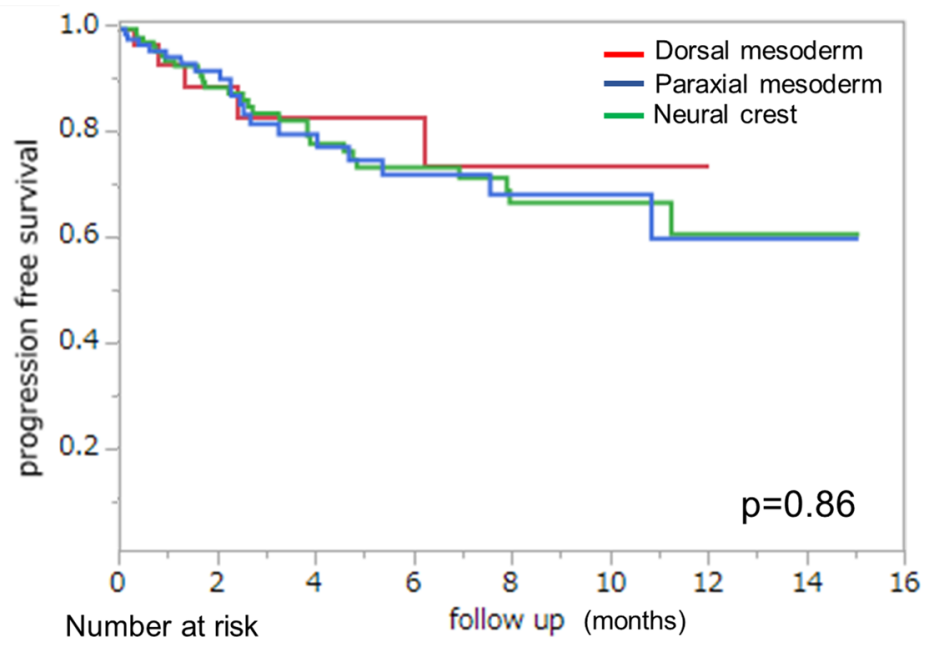

$\begin{array}{lccccccccc}\text { Dorsal mesoderm } & 34 & 19 & 13 & 11 & 8 & 3 & 1 & & \\ \text { Paraxial mesoderm } & 105 & 62 & 37 & 27 & 19 & 14 & 8 & 4 & 1 \\ \text { Neural crest } & 127 & 84 & 55 & 42 & 29 & 15 & 8 & 3 & 2\end{array}$

B

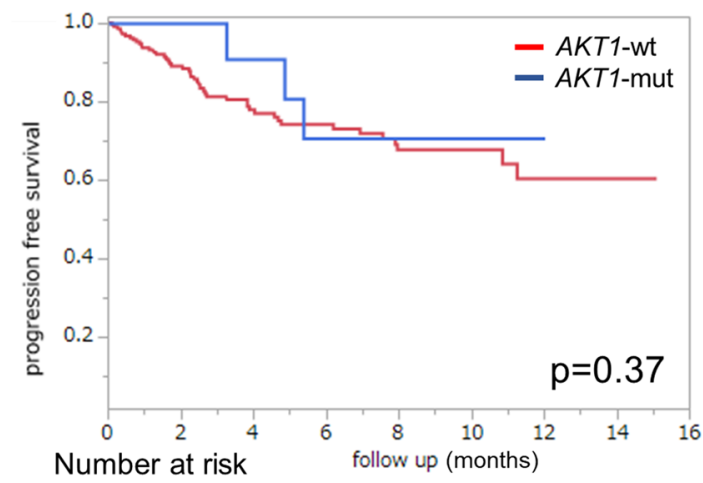

$\begin{array}{llllllllll}\text { AKT1-wt } & 238 & 145 & 90 & 69 & 50 & 27 & 13 & 4 & 1\end{array}$

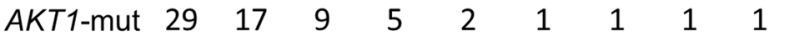

D



$\begin{array}{llllllllll}\text { POLR2A-wt } & 238 & 156 & 96 & 71 & 48 & 24 & 12 & 3 & 1\end{array}$ POLR2A-mut $\begin{array}{lllllllll}17 & 7 & 4 & 4 & 4 & 4 & 2 & 2 & 1\end{array}$

\section{C}

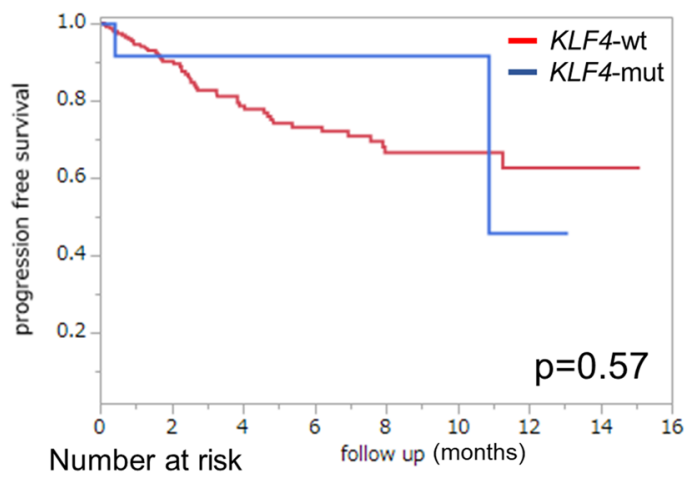

$\begin{array}{llllllllll}K L F 4-w t & 238 & 153 & 94 & 69 & 47 & 25 & 13 & 4 & 1\end{array}$ $\begin{array}{llllllllll}\text { KLF4-mut } & 16 & 9 & 6 & 6 & 5 & 3 & 1 & 1 & 1\end{array}$

$\mathrm{E}$

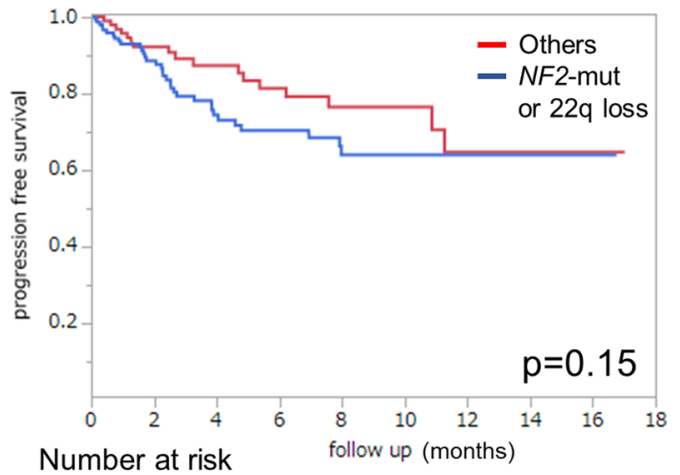

Others

$\begin{array}{lllllllll}111 & 67 & 43 & 36 & 25 & 15 & 6 & 2 & 1\end{array}$

NF2-mut

$\begin{array}{lllllllll}158 & 96 & 57 & 39 & 27 & 13 & 8 & 2 & 1\end{array}$ Or $22 q$ loss

Figure 6. Kaplan-Meier plots of time to recurrence based on genetic mutations. (A) Comparison among tumors originating from the dorsal mesoderm, paraxial mesoderm, and neural crest. No significant differences among the three groups $(p=0.86)$. (B) Comparison between $A K T 1$ mutant and $A K T 1$ wild type. No significant difference between the two groups $(p=0.37)$. (C) Comparison between $K L F 4$ mutant and KLF4 wild type. No significant difference between the two groups $(p=0.57)$. (D) Comparison between POLR2A mutant and $P O L R 2 A$ wild type. Progression-free survival was significantly worse among patients harboring mutant than wild type $P O L R 2 A(p=0.05)$. (E) Comparison between NF2 mutant or 22q loss and other mutation types. No significant difference between the two groups $(p=0.15)$. 


\begin{tabular}{|c|c|c|c|c|c|c|}
\hline & \multicolumn{3}{|l|}{ Univariate } & \multicolumn{3}{|l|}{ Multivariate } \\
\hline & Hazard ratio & $p$ value & $95 \%$ CI & Hazard ratio & $p$ value & $95 \% \mathrm{CI}$ \\
\hline Female & 0.76 & 0.37 & $0.42-1.38$ & & & \\
\hline Age $\geq 65$ & 1.49 & 0.21 & $0.80-2.78$ & & & \\
\hline Simpson grade 1-3 & 0.16 & $9.7 \times 10^{-10}$ & $0.09-0.29$ & 0.21 & $1.9 \times 10^{-6}$ & $0.11-0.39$ \\
\hline MIB-1 LI $\geq 3$ & 3.33 & $1.1 \times 10^{-4}$ & $1.81-6.14$ & 1.81 & 0.14 & $0.82-3.98$ \\
\hline Meningothelial & 0.72 & 0.24 & $0.41-1.25$ & & & \\
\hline Transitional & 0.96 & 0.92 & $0.43-2.14$ & & & \\
\hline Fibrous & 0.47 & 0.11 & $0.19-1.19$ & 1.00 & 0.99 & $0.32-3.14$ \\
\hline WHO grade II & 6.44 & $3.9 \times 10^{-9}$ & $3.46-12.0$ & 4.99 & $1.2 \times 10^{-4}$ & $2.20-11.3$ \\
\hline Neural crest & 1.00 & 0.99 & $0.58-1.75$ & & & \\
\hline Paraxial mesoderm & 1.07 & 0.81 & $0.61-1.90$ & & & \\
\hline Dorsal mesoderm & 0.83 & 0.70 & $0.33-2.10$ & & & \\
\hline AKT1 mutation & 0.60 & 0.39 & $0.19-1.94$ & & & \\
\hline KLF4 mutation & 0.67 & 0.55 & $0.11-2.15$ & & & \\
\hline POLR2A mutation & 2.42 & 0.09 & $0.83-5.58$ & 4.08 & $1.7 \times 10^{-2}$ & $1.28-13.0$ \\
\hline NF2 mutation or $22 \mathrm{q}$ loss & 1.48 & 0.18 & $0.84-2.72$ & 1.81 & 0.15 & $0.81-4.04$ \\
\hline
\end{tabular}

Table 2. Risk factors for tumor recurrence. $L I$ indicates labelling index, ST-med supra-tentorial-medial, ST-ant/lat supra-tentorial-antero-lateral, ST-post/lat supra-tentorial-postero-lateral, CI confidence interval.

largely derived from the neural crest ${ }^{24}$. However, the border line of these embryological contributions is different depending on the species investigated ${ }^{23}$. Overall, these studies indicated that the central skull base is derived from the paraxial mesoderm and the posterior skull base originates from the dorsal mesoderm. The lateral region, including the sphenoid wing, is derived from the neural crest and the anterior skull base does not have a clear border; however, a narrower transitional zone between paraxial mesoderm and neural crest is formed ${ }^{23,24}$.

We evaluated the association between tumor location and pathological diagnoses by defining locations in accordance with the embryological origin of the meninges. The tumors in the neural crest originating regions were associated with more frequent fibrous and WHO grade II meningiomas. However, the tumors in the paraxial mesoderm originating locations were associated with more frequent meningothelial meningioma. It was difficult to evaluate meningiomas originating from the dorsal mesoderm owing to the small patient cohort herein; however, tendencies were similar to those of neural crest origin area. The association between tumor location and histopathology might be based on embryonic dural development. Further studies are required to prove this hypothesis.

This study showed the association between genetic alterations and tumor locations consistent with the previous reports ${ }^{8,12,15,29}$. From the embryological viewpoint, we found that the meningiomas harboring AKT1, SMO, KLF4, or POLR2A mutations were significantly associated with paraxial mesodermal origin. However, meningiomas with NF2 mutations or 22q loss were significantly associated with neural crest and dorsal mesoderm origin.

Previous study have reported that sensitivity to NF2 loss-of-function mutations differ between mesodermderived and neural crest-derived meninges in transgenic mice ${ }^{30}$. Recently, Boetto et al. ${ }^{26}$ reported that selective sensitivity of the skull-base arachnoid to SMO activation initiated meningothelial meningioma in the transgenic mice. These results may explain the differences in genetic status and pathology in accordance with tumor location. However, further biological studies on humans are necessary.

This study indicates associations between driver mutations and histological findings, consistent with previous reports $^{12,15}$. The proportion of meningothelial meningiomas harboring AKT1, KLF4, SMO, or POLR2A mutations was significantly high. Fibrous and WHO grade II tumors primarily harbored an NF2 mutation or 22q loss.

This study, along with previous studies, clearly suggests that among WHO grade I meningiomas, the genetic background of the fibrous type differed from that of meningothelial and transitional types ${ }^{12,29}$. Previous studies have reported that in a transgenic mouse, meningothelial meningiomas originated from arachnoid barrier cells and fibrous meningiomas originated from dural border cells ${ }^{26,30}$. Apparently, these cells differ in their sensitivity to specific genetic mutations, depending on the tumor location ${ }^{26,30,31}$. These results further corroborate the present association between pathological diagnoses and mutations.

This study shows that the POLR2A mutation is a potentially suitable marker for meningiomas with poor prognoses, especially among WHO grade I skull-base meningiomas. Furthermore, the POLR2A mutation was most frequently observed in the central region, which is originated from the paraxial mesoderm. POLR2A is located at 17p13.1 and encodes RNA polymerase II, which plays a fundamental role in eukaryotic organisms. The detailed biological role of the POLR2A mutation in meningiomas remains currently unknown ${ }^{32}$. An RNA polymerase inhibitor, alpha-amanitin, is reported to suppress colorectal tumors harboring the POLR2A mutation ${ }^{33}$. Alpha-amanitin could be a candidate for molecular targeted therapy for the meningiomas harboring POLR2A mutation. The clinical and biological characteristics of meningiomas harboring POLR2A mutation warrant further clarification.

One limitation of our study is its retrospective, single-center design. Another limitation would be that we analyzed a limited number of genetic mutations. Mutations in the genes analyzed herein are frequent; however, other 
mutations including those in TRAF7, hTERT, SMARCB1, SUFU, and PIK3CA also occur in meningiomas ${ }^{15,34-37}$. Moreover, tumorigenesis in meningiomas is associated with not only these driver genetic mutations but also global gene expression profiles and methylation status ${ }^{38}$. This study analyzed genes with hotspot point mutations through Sanger Sequencing and 22q loss through microsatellite analysis. We speculate that these genes can be easily analyzed in the clinical setting. To completely elucidate the association between meningioma tumorigenesis and the embryological origin of the meninges, comprehensive genetic analysis including that of global expression profiles and methylation status is necessary.

\section{Conclusion}

This study shows that meningiomas, according to the embryologic origin of their dural attachment, display differences in pathological diagnosis and genetic abnormalities. Furthermore, this study is the first to show that the POLR2A mutation is a potential indicator of increased tumor recurrence. Assessment of the embryological origin of the meninges may provide novel insights into the pathomechanism of meningiomas. Future molecular biological studies on meningeal embryology are necessary.

\section{Materials and methods}

All methods were carried out in accordance with relevant guidelines and regulations.

Patient population. This study was approved by the Institutional Review Board of the University of Tokyo (Approval Number G10028), and informed consent in writing was obtained from all subjects. We retrospectively analyzed data on 499 patients who underwent resection of meningiomas at the University of Tokyo Hospital between January 2000 and June 2017. We excluded 153 patients from whom fresh-frozen specimens or tumor DNA were not obtained. When the patient underwent multiple surgeries, data from only the first surgery were used. Sixty-eight patients having undergone previous tumor resection at another hospital were excluded. Furthermore, 2 patients harboring NF2, 2 patients having undergone radiation therapy before surgery, and 5 patients with multiple meningiomas were excluded from the study. Finally, the study included 269 patients.

Data collection. We evaluated the following parameters: sex, age, location of origin (attachment to dura), pathological diagnosis, extent of resection (Simpson grade), the need for additional treatment (surgery or/and radiosurgery), and time to tumor recurrence by reviewing the clinical and surgical records. Location was initially defined in accordance with the existing surgical classification based on the anatomical location of tumor dural attachment, in order to accurately extract data from these records. Thus, we classified supra-tentorial locations on the convexity, falx, and parasagittal areas into three types: supra-tentorial-medial (ST-med), supra-tentorialantero-lateral (ST-ant/lat), and supra-tentorial-postero-lateral (ST-post/lat). The border between the "anterior" and "posterior" convexities was the coronal suture. Furthermore, the skull-base lesions were classified into four locations: anterior, central, posterior, and lateral. "Anterior" lesions included the anterior cranial fossa, olfactory groove, and planum sphenoidale. "Central" lesions included the anterior clinoid process, posterior clinoid process, tuberculum sellae, Meckel's cave, cavernous sinus, clival, petroclival-anterior to internal auditory meatus (IAM), and cerebellopontine angle (CPA)-anterior to IAM. "Lateral" lesions included the sphenoid wing and the tentorial attachments, extending into middle cranial fossa, and all middle fossa lesions. "Posterior" included the foramen magnum, CPA posterior to IAM, jugular foramen, cerebellar convexity, and tentorial-extending to the posterior fossa (Fig. 1). We classified the cases, which occupied broader areas, in accordance with the area of the most extensive attachment.

We defined the locations of tumor origin on the basis of the existing knowledge of the embryological origin of leptomeninges ${ }^{20-22,24,27}$. We generated a scheme of normal meningeal development in accordance with their origin (Supplemental Fig. 7). Furthermore, we defined the embryological origins of anatomical locations of the meninges as follows: neural crest origin, including "Lateral," "ST-med," "ST-ant/lat," and "Cerebellar tentorium"; paraxial mesodermal origin, including the "Anterior," "Central," and "ST-post/lat"; dorsal mesodermal, including the "Posterior" group.

Patients were followed-up through contrast-enhanced magnetic resonance imaging (CE-MRI) at $2 \mathrm{~d}$, 6 months, and 1 year after surgery. If there no tumor recurrence was observed, follow-up was regularly continued every year through CE-MRI. For MRI in all cases, we conducted a central review. The precise locations of the tumor origin were defined through preoperative MR images through inter-observer agreement between the neuro-radiologist and two neurosurgeons blinded to the clinical or genetic data. Furthermore, we defined tumor recurrence through inter-observer agreement between the neuro-radiologist and two neurosurgeons blinded to the clinical or genetic data, on the detection of apparent enlargement of residual tumors on CE-MRI.

We conducted a central review of all pathological diagnoses for cases in accordance with the 2016 WHO Classifications of Tumors of the Central Nervous System including the cases diagnosed in the basis of 2000 or 2007 WHO Classifications of Tumors of the Central Nervous System. Transitional meningioma was defined on the basis of the 2016 WHO Classifications of Tumors of the Central Nervous System as a WHO grade I meningioma characterized by the coexistence of meningothelial cells and fibrous architectural patterns. The MIB-1 LI was determined using the highest LI values in areas of their maximum density as identified through visual analysis.

DNA extraction and Sanger sequencing. Tumor DNA was extracted from fresh-frozen tumors, using the QIAmp DNA minikit (QIAGEN; Venlo, Netherlands) in accordance with the manufacturer's instructions, and DNA quality was evaluated using a spectrophotometer. We sequenced mutational hotspots of each gene 
except NF2. Mutations in AKT1 (c.49G > A [p.Glu17Lys]), KLF4 (c.1228A > C [p.Lys409Gln]), SMO (c.1234C > T [p.Leu412Phe] and c.1604G > T [p.Trp535Leu]), and POLR2A (c.1207C > A [p.Gln403Lys] or c.1310-1315 del ACCTTC [p.Leu438_His439del]) were screened through direct Sanger sequencing in all cases. The primers were designed using Primer3. Since NF2 has no mutational hotspots, we performed direct Sanger sequencing for all exons, using the primers generated from the exon primer. For PCR, 50 ng of DNA and KOD FX NEO were used. PCR was performed with $20 \mu \mathrm{l}$ reaction mixtures and the following reaction cycles: initial denaturation at $94{ }^{\circ} \mathrm{C}$ for $2 \mathrm{~min}$, followed by 32 cycles with denaturation at $98^{\circ} \mathrm{C}$ for $10 \mathrm{~s}$, annealing at $58-60^{\circ} \mathrm{C}$ for $30 \mathrm{~s}$, and extension at $68^{\circ} \mathrm{C}$ for $30 \mathrm{~s}$, followed by final extension at $68^{\circ} \mathrm{C}$ for $7 \mathrm{~min}$. Sequences were determined using an ABI 3130xl Genetic Analyzer (Applied Biosystems).

Microsatellite analysis. We performed microsatellite analysis to detect $22 \mathrm{q}$ Loss. This analysis aimed to compare germline and tumor DNA, using both blood and tumor samples. In our study, blood samples were obtained from 241 of the 269 patients. We used the following five microsatellite polymorphic markers franking NF2, selected from the Genome Data Base: D22S268, D22S1163, D22S929, D22S280, and D22S282. The sense primer was labeled with a fluorescent dye, and PCR was performed for $25-30$ cycles at $58-60{ }^{\circ} \mathrm{C}$ for annealing, using the Gene Amp 9700 Thermal Cycler (PE Biosystems; Framingham, Massachusetts, USA). PCR products were separated through capillary electrophoresis with the Genetic Analyzer 310, and the analysis was performed using the Gene Scan Program (PE Biosystems) ${ }^{39}$.

Statistical analysis. To determine the association between tumor location and pathological diagnosis and between tumor location and genetic mutational status, the chi-square test was used. For multiple comparisons, Bonferroni correction was applied. Progression-free survival was defined as the time between surgery and recurrence or final follow-up. Recurrence-free cases were censored on final follow-up. Kaplan-Meier survival curves were plotted and differences in progression-free survival between groups were compared using the log-rank test. We assessed the effect of sex, age, Simpson grade, MIB-1 LI, pathological diagnosis, embryological tumor localization, and mutational status through univariate analyses with a Cox proportional hazards model. Thereafter, multivariate analysis was performed using parameters with a $p$ value of $<0.2$ on univariate analysis. All statistical analyses were performed with JMP Pro version 11 (SAS Institute, Inc.; Cary, North Carolina, USA). A $p$ value of $<0.05$ was considered statistically significant. We excluded $S M O$ mutation from the evaluating factor since there was only 1 case with SMO mutation.

\section{Data availability}

Data are available on reasonable request. The authors confirm that the data supporting the findings of this study will be shared by request from any qualified investigator.

Received: 10 June 2020; Accepted: 15 March 2021

Published online: 26 March 2021

\section{References}

1. Bulleid, L. S., James, Z., Lammie, A., Hayhurst, C. \& Leach, P. A. The effect of the revised WHO classification on the incidence of grade II meningioma. Br. J. Neurosurg. https://doi.org/10.1080/02688697.2019.1639616 (2019).

2. Sade, B. et al. World Health Organization Grades II and III meningiomas are rare in the cranial base and spine. Neurosurgery $\mathbf{6 1}$, 1194-1198. https://doi.org/10.1227/01.neu.0000306097.38141.65 (2007).

3. Cornelius, J. F. et al. Malignant potential of skull base versus non-skull base meningiomas: clinical series of 1,663 cases. Acta Neurochir. 155, 407-413. https://doi.org/10.1007/s00701-012-1611-y (2013).

4. McGovern, S. L. et al. A comparison of World Health Organization tumor grades at recurrence in patients with non-skull base and skull base meningiomas. J. Neurosurg. 112, 925-933. https://doi.org/10.3171/2009.9.jns09617 (2010).

5. Kane, A. J. et al. Anatomic location is a risk factor for atypical and malignant meningiomas. Cancer 117, 1272-1278. https://doi. org/10.1002/cncr.25591 (2011).

6. Kros, J. et al. NF2 status of meningiomas is associated with tumour localization and histology. J. Pathol. 194, 367-372. https://doi. org/10.1002/path.909 (2001).

7. Riemenschneider, M. J., Perry, A. \& Reifenberger, G. Histological classification and molecular genetics of meningiomas. Lancet Neurol. 5, 1045-1054. https://doi.org/10.1016/S1474-4422(06)70625-1 (2006).

8. Clark, V. E. et al. Genomic analysis of non-NF2 meningiomas reveals mutations in TRAF7, KLF4, AKT1, and SMO. Science 339, 1077-1080. https://doi.org/10.1126/science.1233009 (2013).

9. Williams, S. R. et al. Genomic analysis of posterior fossa meningioma demonstrates frequent AKT1 E17K mutations in foramen magnum meningiomas. J. Neurol. Surg. B Skull Base 80, 562-567. https://doi.org/10.1055/s-0038-1676821 (2019).

10. Juratli, T. A. et al. DMD genomic deletions characterize a subset of progressive/higher-grade meningiomas with poor outcome. Acta Neuropathol. 136, 779-792. https://doi.org/10.1007/s00401-018-1899-7 (2018).

11. Strickland, M. R. et al. Targeted sequencing of SMO and AKT1 in anterior skull base meningiomas. J. Neurosurg. 127, 438-444. https://doi.org/10.3171/2016.8.Jns161076 (2017).

12. Brastianos, P. K. et al. Genomic sequencing of meningiomas identifies oncogenic SMO and AKT1 mutations. Nat. Genet. 45, 285-289. https://doi.org/10.1038/ng.2526 (2013).

13. Sahm, F. et al. AKT1E17K mutations cluster with meningothelial and transitional meningiomas and can be detected by SFRP1 immunohistochemistry. Acta Neuropathol. 126, 757-762. https://doi.org/10.1007/s00401-013-1187-5 (2013).

14. Bi, W. L. et al. Genomic landscape of intracranial meningiomas. J. Neurosurg. 125, 525-535. https://doi.org/10.3171/2015.6.Jns15 591 (2016).

15. Clark, V. E. et al. Recurrent somatic mutations in POLR2A define a distinct subset of meningiomas. Nat. Genet. 48, 1253-1259. https://doi.org/10.1038/ng.3651 (2016).

16. Wellenreuther, R. et al. Analysis of the neurofibromatosis 2 gene reveals molecular variants of meningioma. Am. J. Pathol. 146, 827-832 (1995). 
17. Hartmann, C. et al. NF2 mutations in secretory and other rare variants of meningiomas. Brain Pathol. 16, 15-19 (2006).

18. Reuss, D. E. et al. Secretory meningiomas are defined by combined KLF4 K409Q and TRAF7 mutations. Acta Neuropathol. 125, 351-358. https://doi.org/10.1007/s00401-013-1093-x (2013).

19. Youngblood, M. W. et al. Correlations between genomic subgroup and clinical features in a cohort of more than 3000 meningiomas. J. Neurosurg. https://doi.org/10.3171/2019.8.Jns191266 (2019).

20. O’Rahilly, R. \& Müller, F. The meninges in human development. J. Neuropathol. Exp. Neurol. 45, 588-608 (1986).

21. Dasgupta, K. \& Jeong, J. Developmental biology of the meninges. Genesis 57, e23288. https://doi.org/10.1002/dvg.23288 (2019).

22. Jiang, X., Iseki, S., Maxson, R. E., Sucov, H. M. \& Morriss-Kay, G. M. Tissue origins and interactions in the mammalian skull vault. Dev. Biol. 241, 106-116. https://doi.org/10.1006/dbio.2001.0487 (2002).

23. Noden, D. M. \& Trainor, P. A. Relations and interactions between cranial mesoderm and neural crest populations. J. Anat. 207, 575-601. https://doi.org/10.1111/j.1469-7580.2005.00473.x (2005).

24. McBratney-Owen, B., Iseki, S., Bamforth, S. D., Olsen, B. R. \& Morriss-Kay, G. M. Development and tissue origins of the mammalian cranial base. Dev. Biol. 322, 121-132. https://doi.org/10.1016/j.ydbio.2008.07.016 (2008).

25. Wu, T., Chen, G., Tian, F. \& Liu, H. X. Contribution of cranial neural crest cells to mouse skull development. Int. J. Dev. Biol. 61, 495-503. https://doi.org/10.1387/ijdb.170051gc (2017).

26. Boetto, J., Apra, C., Bielle, F., Peyre, M. \& Kalamarides, M. Selective vulnerability of the primitive meningeal layer to prenatal Smo activation for skull base meningothelial meningioma formation. Oncogene 37, 4955-4963. https://doi.org/10.1038/s41388-0180328-7 (2018).

27. Tanaka, M. Embryological consideration of dural AVF. Acta Neurochir. Suppl. 123, 169-176. https://doi.org/10.1007/978-3-31929887-0_24 (2016).

28. Rai, R., Iwanaga, J., Shokouhi, G., Oskouian, R. J. \& Tubbs, R. S. The tentorium cerebelli: a comprehensive review including its anatomy, embryology, and surgical techniques. Cureus 10, e3079. https://doi.org/10.7759/cureus.3079 (2018).

29. Yuzawa, S., Nishihara, H. \& Tanaka, S. Genetic landscape of meningioma. Brain Tumor Pathol. 33, 237-247. https://doi.org/10. 1007/s10014-016-0271-7 (2016).

30. Kalamarides, M. et al. Identification of a progenitor cell of origin capable of generating diverse meningioma histological subtypes. Oncogene 30, 2333-2344. https://doi.org/10.1038/onc.2010.609 (2011).

31. Peyre, M. et al. PDGF activation in PGDS-positive arachnoid cells induces meningioma formation in mice promoting tumor progression in combination with Nf2 and Cdkn2ab loss. Oncotarget 6, 32713-32722. https://doi.org/10.18632/oncotarget.5296 (2015).

32. Wintzerith, M., Acker, J., Vicaire, S., Vigneron, M. \& Kedinger, C. Complete sequence of the human RNA polymerase II largest subunit. Nucleic Acids Res. 20, 910. https://doi.org/10.1093/nar/20.4.910 (1992).

33. Liu, Y. et al. TP53 loss creates therapeutic vulnerability in colorectal cancer. Nature 520, 697-701. https://doi.org/10.1038/natur e14418 (2015).

34. Goutagny, S. et al. High incidence of activating TERT promoter mutations in meningiomas undergoing malignant progression. Brain Pathol. 24, 184-189. https://doi.org/10.1111/bpa.12110 (2014).

35. Sahm, F. et al. TERT promoter mutations and risk of recurrence in meningioma. J. Natl. Cancer Inst. https://doi.org/10.1093/jnci/ djv377 (2016).

36. Youngblood, M. W. et al. Associations of meningioma molecular subgroup and tumor recurrence. Neuro Oncol. https://doi.org/ 10.1093/neuonc/noaa226 (2020).

37. Abedalthagafi, M. et al. Oncogenic PI3K mutations are as common as AKT1 and SMO mutations in meningioma. Neuro Oncol. 18, 649-655. https://doi.org/10.1093/neuonc/nov316 (2016).

38. Sahm, F. et al. DNA methylation-based classification and grading system for meningioma: a multicentre, retrospective analysis. Lancet Oncol. 18, 682-694. https://doi.org/10.1016/s1470-2045(17)30155-9 (2017).

39. Ueki, K., Wen-Bin, C., Narita, Y., Asai, A. \& Kirino, T. Tight association of loss of merlin expression with loss of heterozygosity at chromosome 22q in sporadic meningiomas. Cancer Res. 59, 5995-5998 (1999).

\section{Acknowledgements}

We thank Dr. Kostadin Karagiozov for his support and help the English proofreading. This study was financially supported by Grant-in-aid for Scientific Research (B) (No. 17H04301 to N.S.) from the Japan Society for the Promotion of Science, Grant-in-aid for Scientific Research (C) (No. 19K09499 to H.N.) from the Japan Society for the Promotion of Science. Grant-in-aid for Scientific Research (C) (No. 19K09473 to S.M.) from the Japan Society for the Promotion of Science and Research grant from Takeda Science Foundation (to S.M.).

\section{Author contributions}

Study design: A.O., S.M., H.N., N.S. Acquisition of data: A.O., H.H., S.D., Y.T., M.S. Analysis of data: A.O., M.S., J.M., M.T. Drafting of manuscript: A.O., M.S., H.N., N.S. All authors have read and approved the final manuscript.

\section{Competing interests}

The authors declare no competing interests.

\section{Additional information}

Supplementary Information The online version contains supplementary material available at https:/doi.org/ 10.1038/s41598-021-86298-9.

Correspondence and requests for materials should be addressed to S.M.

Reprints and permissions information is available at www.nature.com/reprints.

Publisher's note Springer Nature remains neutral with regard to jurisdictional claims in published maps and institutional affiliations. 
(c) (i) Open Access This article is licensed under a Creative Commons Attribution 4.0 International cc) License, which permits use, sharing, adaptation, distribution and reproduction in any medium or format, as long as you give appropriate credit to the original author(s) and the source, provide a link to the Creative Commons licence, and indicate if changes were made. The images or other third party material in this article are included in the article's Creative Commons licence, unless indicated otherwise in a credit line to the material. If material is not included in the article's Creative Commons licence and your intended use is not permitted by statutory regulation or exceeds the permitted use, you will need to obtain permission directly from the copyright holder. To view a copy of this licence, visit http://creativecommons.org/licenses/by/4.0/.

(C) The Author(s) 2021 